\title{
Evaluación de diferentes valores de referencia espirométricos para el diagnóstico de alteración restrictiva en población chilena
}

\author{
MÓNICA GUTIÉRREZ C., ANA MARÍA DEL FIERRO O., \\ ROBERTO VALLEJO P. y CARINA FACCILONGO G.
}

\begin{abstract}
Assessment of different spirometric reference values for diagnosing restrictive impairment in chilean population
\end{abstract}

In 1988, the Chilean Respiratory Society decided to get national spirometric reference values $(S R V)$, because those used at that time (Knudson et al) underestimated the values in our population. Since then, and using national SRV, it has been observed an increase of the positive false restrictive impairment diagnoses. Our aim was to analyse the probability of making a restrictive impairment diagnosis, according to different spirometric reference values, using the total lung capacity (TLC) measurement as a gold standard. Patients and Methods: We analysed the spirometries from male and female patients older than 59 y.o., in whom lung volumes by helium dilution, had been measured concurrently in our laboratory during 2004. Restrictive impairment was substantiated (confirmed) if TLC was lower than its theoretical value (European Community) minus 1,64. RSD (standard deviation of the residual). Spirometries from the same patients were analysed with different SRV and several statistical indicators of diagnostic tests were calculated. Results: 91 patients (mean age: 72 y.o. ranging from 60 to 89) were assessed. We confirmed a restrictive impairment in 35 of them (38\%). Using national SRV, sensitivity (S) was $86 \%$, specificity (E) $59 \%$ and the post-test probability (PPT) 57\%. Using NHANES III SRV, S was 54\%, E was $91 \%$ and PPT 79\% Using Knudson SRV S was 49\%, E 93\% and PPT 81\%, while by using Platino study SRV, S was of 49\%, E was 77\% and PPT 57\%. Conclusion: Similarly to previous reports, we have found an important difference in the probability of diagnosing restriction in our population, according to the SRV used.

Key words: spirometry, reference values, FVC, lung volumes, total lung capacity, restrictive impairment.

\section{Resumen}

A finales de los años 80 se planteó la necesidad de disponer de valores de referencia espirométricos (VRE) nacionales, ya que los utilizados en esa época (Knudson) subestimaban los valores en nuestra población. Posteriormente, al utilizar VRE nacionales, se ha observado un aumento de los diagnósticos de restricción falsos positivos. El objetivo de este trabajo fue analizar la probabilidad de hacer el diagnóstico de restricción a través de la espirometría, según diferentes valores de referencia utilizando como gold standard la medición de la capacidad pulmonar total (CPT). Material y Método: Se analizaron las espirometrías de pacientes mayores de 59 años, estudiados además con volúmenes pulmonares por técnica de dilución de helio durante el año 2004. Como criterio de restricción se utilizó el valor inferior a CPT teórica menos 1,64. RSD (desviación standard del residuo) con respecto a los valores teóricos de la comunidad europea. Se analizaron las espirometrías de los mismos pacientes con diferentes VRE y se calcularon diferentes indicadores estadísticos de pruebas diagnósticas. Resultados: Se evaluaron 91 casos con edad promedio de 72 años (rango 60 a 89) encontrando verdadera restricción en 35 de ellos (38\%). Al utilizar VRE

* Hospital Naval Viña del Mar, Facultad de Medicina, Universidad de Valparaíso. 
nacionales la sensibilidad (S) fue de $86 \%$, la especificidad (E) de $59 \%$ y la probabilidad postest (PPT) de 57\%. Con NHANES III la S fue de 54\%, la E de $91 \%$ y la PPT de 79\%.Con Knudson la $S$ fue de $49 \%$, la E de $93 \%$ y la PPT de $81 \%$ mientras que con Platino la S fue de 49\%, la E de 77\% y la PPT de 57\%. Se concluye que existe una gran diferencia en la probabilidad de diagnosticar restricción según el VRE utilizado, de manera similar a lo publicado por otros autores.

Palabras clave: Espirometría, valores de referencia, CVF, volúmenes pulmonares, capacidad pulmonar total, limitación restrictiva.

\section{Introducción}

En la actualidad la espirometría es una herramienta muy importante en la medicina moderna. Se ha demostrado su utilidad en el diagnóstico y el control de la evolución de las enfermedades pulmonares, la evaluación preoperatoria, la estimación del estado basal de personas expuestas a sustancias dañinas, la detección de reacciones adversas a medicamentos con toxicidad pulmonar conocida, la cuantificación de la incapacidad laboral y en estudios epidemiológicos ${ }^{1-3}$. En los últimos años se ha incentivado su uso a nivel de atención primaria en la campaña de prevención y detección precoz de la Enfermedad Pulmonar Obstructiva Crónica (EPOC) relacionada con el tabaco ${ }^{4-6}$.

Por lo anterior, la interpretación de este examen es crucial, ya que se toman y respaldan varias decisiones de acuerdo a sus resultados. Para la correcta interpretación se requiere la comparación de los resultados obtenidos con lo considerado "normal" para una persona sana de igual sexo, edad y estatura ${ }^{7}$. Para definir esta "normalidad" se han realizado varios estudios epidemiológicos que miden los parámetros espirométricos en una muestra representativa de la población sana, no fumadora de diferentes comunidades, habiéndose demostrado que hay diferencias entre diversos grupos étnicos ${ }^{8-12}$.

En Chile, a finales de los años 80 se planteó la necesidad de contar con valores de referencia espirométricos (VRE) nacionales, ya que los utilizados a partir del año 1988, como recomendación de la Sociedad Chilena de Enfermedades Respiratorias (SER) ${ }^{13,14}$ eran los de Knudson y cols $^{15}$ que subestimaban los valores de nuestra población ${ }^{16-19}$. A partir del año 1997 se han utilizado VRE nacionales ${ }^{20,21}$ con lo cual hemos observado un aumento de los diagnósticos "restrictivos", especialmente en pacientes mayores de 60 años, cuestionándose su uso ${ }^{22-24}$.

El objetivo de este trabajo fue analizar con cual VRE la interpretación de "restricción" se acerca más a la verdad, al utilizar el diagnóstico de "restricción" realizado en base a la medición de la Capacidad Pulmonar Total (CPT).

\section{Pacientes y Método}

Se revisaron todos los estudios de función pulmonar realizados durante el año 2004 a pacientes mayores de 59 años en el laboratorio de función pulmonar del Hospital Naval de Viña del Mar, en que se hubiese realizado en la misma sesión espirometría y volúmenes pulmonares medidos por técnica de dilución de helio, que cumplían con las normas de la ATS (American Thoracic Society) $)^{25}$ para su realización. Estas espirometrías fueron interpretadas de acuerdo a varias ecuaciones de referencia que se han utilizado o serían aplicables en teoría a nuestra población:

1. Gutiérrez y cols $1996^{20,21}$ (VRE nacionales) Ecuaciones de regresión lineal obtenidas de personas chilenas sanas no fumadoras elegidas al azar y representativas de la población de la V Región. Para los hombres adultos se derivó una ecuación a partir de 150 personas con rango de edad de 19 a 80 años, promedio de edad $=38,4$ años con 27 hombres mayores de 49 años (18\%). Para las mujeres se derivó una ecuación a partir de 255 personas entre 21 y 79 años, promedio de edad $=$ 38,8 años, con 57 personas sobre 49 años (22\%).

2. NHANES III ${ }^{9}$ : Ecuaciones de regresión polinomiales obtenidas de población mejicano-americana de Estados Unidos. Se analizaron 506 hombres de 20 a 80 años de los cuales 89 eran mayores de 49 años (18\%) y 872 mujeres de 18 a 80 años con 203 de ellas sobre 50 años (23\%).

3. Knudson y cols ${ }^{15}$ : Ecuaciones de regresión lineal obtenidas de población sana no fumadora americana, no mejicano-americana, de Tucson Arizona. Para los hombres adultos se derivó una ecuación a partir de 86 personas de 25 a 85. Para las mujeres se derivaron dos ecuaciones, una a partir de 176 personas entre 20 y 69 años y otra, a partir de 28 mujeres de 70 a 88 años.

4. PLATINO ${ }^{26}$ : Ecuaciones de regresión lineal obtenidas de población sana no fumadora, de 5 ciudades latinoamericanas. Se estudió un 
total de 566 hombres mayores de 40 años, con promedio de edad de 54,0 años y 954 mujeres mayores de 40 años, con promedio de edad de 55,4 años.

Se utilizó como criterio espirométrico de "Restricción" una relación $\mathrm{VEF}_{1} / \mathrm{CVF}$ superior al percentil $5\left(\mathrm{P}_{5}\right)$ de este parámetro, con capacidad vital forzada $(\mathrm{CVF})$ inferior al $\mathrm{P}_{5}$. Para la interpretación de los volúmenes pulmonares se utilizaron las ecuaciones publicadas por la Sociedad Europea de Respiratorio (ERS) ${ }^{27}$ definiendo como "restrictivo" una CPT medida inferior al valor $\mathrm{P}_{5}$ calculado para ese parámetro. Para los cálculos estadísticos se definieron 2 grupos: los sujetos "restrictivos" y los "no restrictivos" que comprendieron a todos los que no cumplían los criterios antes definidos. Se utilizaron los siguientes indicadores estadísticos de test diagnósticos ${ }^{28}$ :

Sensibilidad: (concordantes restrictivos/total verdaderos restrictivos) 100

Especificidad: (concordantes no restrictivos/ total verdaderos no restrictivos) $\cdot 100$

VPP: (concordantes restrictivos/total restrictivos espirométricos) $\cdot 100$

VPN: (concordantes no restrictivos/total no restrictivos espirométricos) 100

LR +: sensibilidad / ( 1 - especificidad $)$

LR-: (1- sensibilidad) / especificidad

Probabilidad Pre Test $=$ prevalencia $($ Pre $)$

Probabilidad Post Test:

Con test $(+):($ S.Pre $) /((S \cdot P r e)+(1-E)(1-P r e))$

Con Test (-): (1-S)(Pre)/(E·(1-Pre)+(1-S)(Pre))

Además se analizaron todos estos exámenes de acuerdo al informe que se había emitido originalmente, que corresponde a la interpretación manual o médica entregada por el médico a cargo del laboratorio de función pulmonar, que difiere de la anterior en que flexibiliza la interpretación cuando se trata de valores límites agregando términos como: "dentro de límites normales bajos"

\section{Resultados}

Durante el año 2004 se realizaron 431 espirometrías en personas de 60 a 93 años. El análisis de la interpretación según las diferentes ecuaciones se muestra en la Tabla 1 . Se observa una gran variabilidad en los diagnósticos restrictivos desde un $46 \%$ utilizando Gutiérrez $1996^{19,22}$, hasta un $12 \%$ utilizando Knudson ${ }^{15}$.

De estos 431 pacientes, 91 contaban con medición de volúmenes pulmonares realizada con técnica de dilución de helio y cumplían con los requerimientos técnicos de la ERS. Su edad fluctuó entre los 60 y los 89 años, con un promedio de 72 años. Se encontró que 35 casos (38\%) tenían el diagnóstico de "restricción" según CPT. Sólo con VRE nacionales se observó un sobrediagnóstico de restricción, con 53 casos (58\%). PLATINO $^{15}$ fue el que más se acercó al valor real, con 30 casos (33\%), mientras que con Knudson y NAHNES III $^{8}$ se subdiagnosticaron los pacientes restrictivos: 21 casos $(23 \%)$ y 24 casos $(26 \%)$ respectivamente.

A partir de la concordancia o disconcordancia con respecto a la restricción que se muestra en la Tabla 2, se calcularon los indicadores estadísticos que se muestran en la Tabla 3.

\section{Conclusiones}

Queda demostrado que la interpretación de la espirometría depende en forma importante de los valores de referencia utilizados (Tabla 1). Según las normas de la ATS y ERS ${ }^{14}$, debiéramos utilizar aquellos que provengan de poblaciones lo más similares a la que se está estudiando, tanto en el aspecto étnico, como en edad. Al utilizar en nuestra población de mayor edad los VRE nacionales, derivados de población chilena ${ }^{19,23}$ y étnicamente similares, observamos un aumento superior a tres veces de los diagnósticos "restrictivos" y una disminución importante de los diagnósticos "obstructivos"

Tabla 1. Interpretación de espirometrías realizadas el año 2004 a personas de 60 años y más

\begin{tabular}{lrrrrr}
\hline & VRE nacionales $^{\mathbf{2 0 , 2 1}}$ & NHANES III & \multicolumn{1}{c}{ Knudson $^{\mathbf{1 5}}$} & Platino $^{\mathbf{2 6}}$ \\
\hline Normales & $152(35 \%)$ & $252(58 \%)$ & $239(55 \%)$ & $269(62 \%)$ \\
Obstructivos & $80(19 \%)$ & $105(24 \%)$ & $139(32 \%)$ & $79(18 \%)$ \\
Restrictivos & $199(46 \%)$ & $74(17 \%)$ & $53(12 \%)$ & $83(19 \%)$ \\
Total & 431 & 431 & 431 & 431 \\
\hline
\end{tabular}

VRE: Valores de referencia espirométrica. 
Tabla 2. Concordancia entre diagnóstico espirométrico según los diferentes valores de referencia espirométrica y el diagnóstico definitivo de restricción definido como disminución de la capacidad pulmonar total

\begin{tabular}{lcccc}
\hline Casos & VRE nacionales $^{\mathbf{2 0 , 2 1}}$ & NHANES III $^{\mathbf{9}}$ & Knudson $^{\mathbf{1 5}^{\mathbf{2}}}$ & Platino $^{\mathbf{2 6}}$ \\
\hline Falsos positivos (n) & 23 & 5 & 4 & 13 \\
Verdaderos negativos (n) & 33 & 51 & 52 & 43 \\
Verdaderos positivos (n) & 30 & 19 & 17 & 17 \\
Falsos negativos (n) & 5 & 16 & 18 & 18 \\
\hline
\end{tabular}

VRE: Valores de referencia espirométrica.

Tabla 3. Indicadores estadísticos de test diagnósticos según cada valor de referencia espirométrico utilizando como gold standard de restricción la disminución de la CPT

\begin{tabular}{lcccc}
\hline & VRE nacionales $^{\mathbf{2 0 , 2 1}}$ & NHANES III $^{\mathbf{9}}$ & Knudson $^{\mathbf{1 5}}$ & Platino $^{\mathbf{2 6}}$ \\
\hline Sensibilidad & 86 & 54 & 49 & 49 \\
Especificidad & 59 & 91 & 93 & 77 \\
Valor predictivo positivo & 57 & 79 & 81 & 57 \\
Valor predictivo negativo & 87 & 76 & 74 & 70 \\
Likelihood ratio para un resultado (+) & 2,08 & 6,07 & 6,8 & 2,09 \\
Likelihood ratio para un resultado (-) & 0,24 & 0,5 & 0,55 & 0,66 \\
Probabilidad pre-test (prevalencia) & 38 & 38 & 38 & 38 \\
Probabilidad post-test: Test (+) & 57 & 79 & 81 & 57 \\
Probabilidad post-test: Test (-) & 13 & 24 & 26 & 30 \\
\hline
\end{tabular}

VRE: Valores de referencia espirométrica.

comparados con los informes previos (Knudson, Tabla 1). Llama la atención que al utilizar NHANES III ${ }^{8}$, de población mejicano-americana y PLATINO ${ }^{15}$, de población latinoamericana, se obtienen más diagnósticos de "normalidad" que con Knudson.

Para poder definir cual de estas interpretaciones es la correcta, utilizamos como gold estándar de restricción una disminución en la CPT medida con la técnica de dilución de helio por debajo del límite inferior de normalidad para este parámetro. Elegimos utilizar los valores de referencia de las guías ERS, que cuentan con cálculo de límite inferior para cada variable, ya que no disponemos de valores de referencia nacionales o de población latinoamericana. Así encontramos que 35 de los 91 pacientes (38\%) tenían diagnóstico definitivo de restricción. Para analizar la concordancia de diagnósticos (Tabla 2) se calculó la sensibilidad, especificidad, VPP, VPN, LR+, LR- y probabilidad post test para cada VRE (Tabla 3). Este "gold standard" ya ha sido utilizado en varias publicaciones de los últimos años ${ }^{33-35}$. Aarón et al en $1999^{34}$ analizó cuan exacta era la espirometría para predecir un defecto pulmonar de tipo restrictivo al analizar 1831 pacientes con ambos estudios (Tabla 4), observó un bajo valor predictivo positivo y concluyó que la espirometría es muy útil para excluir un defecto restrictivo; sin embargo no lo es para predecirlo. Si revisamos la Tabla 4, vemos que en estas 3 publicaciones hay una tendencia muy similar a la encontrada con los VRE nacionales y que no estábamos acostumbrados a ver previamente ya que subvalorábamos la condición de nuestros pacientes.

El conocer el VPP y VPN de la espirometría para el diagnóstico de restricción, permite mejorar la interpretación clínica frente a cada paciente. Por ejemplo, si utilizando los VRE nacionales la espirometría tiene una alteración de tipo restrictiva, ese paciente tiene el $57 \%$ de probabilidad que realmente lo sea, en cambio si es restrictiva al utilizar los VRE de Knudson tiene 
Tabla 4. Indicadores estadísticos de test diagnósticos según cada valor de referencia espirométrico utilizando como gold standard de restricción la disminución de la CPT

\begin{tabular}{lccc}
\hline & Swanney $^{\mathbf{3 3}}$ & Aaron $^{\mathbf{3 4}}$ & Glady $^{\mathbf{3 5}}$ \\
\hline Sensibilidad & 96 & 68 & 49 \\
Especificidad & 82 & 93 & 93 \\
Valor predictivo positivo & 42 & 58 & 81 \\
Valor predictivo negativo & 99 & 95 & 74 \\
\hline
\end{tabular}

Tabla 5. Interpretación de volúmenes pulmonares y espirometrías según Gutiérrez 1996 informe computacional e interpretación médica

\begin{tabular}{lcccc}
\hline & Volúmenes Pulmonares & Informe computacional & Interpretación médica \\
\hline No Restrictivos & (n) & 56 & 38 & 44 \\
Restrictivos & (n) & 35 & 53 & 47 \\
\hline
\end{tabular}

Tabla 6. Cambios de los indicadores estadísticos según se interprete la espirometría en forma computacional o con criterio médico, de acuerdo a los valores de referencia espirométrica nacionales

\begin{tabular}{lcc}
\hline & Informe computacional & Interpretación médica \\
\hline Sensibilidad & 85,71 & 85,71 \\
Especificidad & 58,92 & 69,64 \\
Valor predictivo positivo & 56,6 & 63,82 \\
Valor predictivo negativo & 86,84 & 88,63 \\
Likelihood ratio para un resultado + & 2,08 & 2,82 \\
Likelihood ratio para un resultado - & 0,24 & 0,2 \\
Probabilidad pre-test (prevalencia) & 38,46 & 38,46 \\
Probabilidad post-test & 56,6 & 63,82 \\
\hline
\end{tabular}

el $81 \%$ de probabilidad de serlo. Por el contrario, si la interpretación es "no restrictiva" utilizando los VRE nacionales ese paciente tiene solo un $13 \%$ de probabilidad de serlo, versus un $26 \%$ si se utilizan los VRE de Knudson.

Otro punto a considerar es que todo el análisis que hemos realizado, se ha basado en una interpretación computacional, la cual no es real, ya que se simplifica y pone un punto de corte arbitrario entre lo normal y anormal para cada uno de los parámetros espirométricos, lo que lleva a error cuando los valores están cercanos a ese punto de corte. Por ejemplo, un cambio en $30 \mathrm{ml}$ en la medición del $\mathrm{VEF}_{1}(1970 \mathrm{ml}$ versus $2.000 \mathrm{ml}$ ) con una CVF de $2840 \mathrm{ml}$ hace que cambie la interpretación espirométrica de obstructiva a restrictiva siempre que el límite inferior de normalidad para $\mathrm{VEF}_{1} / \mathrm{CVF}$ sea de $70 \%$. Al respecto, las normas de interpretación de las pruebas funcionales respiratorias ATS $^{29}$ sugieren interpretar con cautela los valores limítrofes con alguna frase como "inusualmente bajos". Los exámenes que revisamos estaban informados originalmente con los VRE nacionales utilizando estos criterios. Así, podemos observar que con esta interpretación disminuyen los diagnósticos restrictivos (Tabla 5) y aumenta la probabilidad post test de restricción de 57 a 64\% (Tabla 6).

Concluimos que realmente estamos realizan- 
do más interpretaciones espirométricas de tipo restrictiva al utilizar los VRE nacionales que con los de Knudson; sin embargo con los VRE nacionales la sensibilidad y especificidad para predecir restricción es muy similar a lo publicado recientemente en la literatura. El bajo VPP de este examen para restricción, pone de manifiesto la importancia de tener en cuenta los antecedentes clínicos o probabilidad pretest para la correcta interpretación de los resultados espirométricos frente a cada paciente y la necesidad de utilizar otros exámenes para asegurar una alteración de tipo restrictivo.

\section{Agradecimientos}

Los autores agradecen al Dr. Claudio Puebla por su revisión y ayuda en la interpretación de los indicadores estadísticos.

\section{Bibliografía}

1.- ATS Snowbird workshop on standardization of spirometry. Am Rev Respir Dis 1979; 119: 831.

2.- ATS Standardization of spirometry: 1987 update. Am Rev Respir Dis 1987; 136: 1285-98.

3.- American Association for Respiratory Care Clinical Practice Guideline: Spirometry, 1996 update. Respir Care 1996; 41: 629-36.

4.- British Thoracic Society guidelines for the management of chronic obstructive pulmonary disease. The COPD Guidelines Group of the Standards of Care Committee of the BTS. Thorax 1997; 52: S1-28.

5.- Global Strategy for the Diagnosis, Management, and Prevention of Chronic Obstructive Pulmonary Disease (GOLD). Am J Respir Crit Care Med 2001; 163: 125676.

6.- FERGUSON G T, ENRIGHT P L, BUIST A S, HIGGINS M W. Office spirometry for lung health assessment in adults: a consensus statement from the National Lung Health Education Program. Respir Care 2000; 45: 513-30.

7.- CRAPO $\mathrm{R}$ O. The role of reference values in interpreting lung function tests. Eur Respir J 2004, 24: 341-2.

8.- ENRIGHT P L, KRONMAL R A, HIGGINS M, SCHENKER M, HAPONIK E F. Spirometry reference values for women and men 65 to 85 years of age. Cardiovascular Health Study. Am Rev Respir Dis 1993 , 147: $125-33$

9.- HANKINSON J, ODENCRANTZ J, FEDAN K. Spirometric Reference Values from a Sample of the General U.S. Population. Am J Respir Crit Care Med 1999; 159: 179-87.

10.- KOROTZER B, ONG S, HANSEN J E. Ethnic differences in pulmonary function in healthy nonsmoking Asian-Americans and European-Americans. Am J Respir Crit Care Med 2000; 161: 1101-8.

11.- MC DONNELL W F, ENRIGHT P L, ABBEY D E, et al. Spirometry reference equations for older adults. Respir Med 1998; 92: 914.
12.- HARIK-KHAN R I, FLEG J L, MULLER D C, WISE $\mathrm{R}$ A. The effect of anthropometric and socioeconomic factors on the racial difference in lung function. Am J Respir Crit Care Med 2001; 164: 1647-54.

13.- MORENO R, OYARZÚN M. Recomendaciones sobre informe espirométrico. Primera parte. Enf Respir Cir Torác 1988; 3: 97-103.

14.- MORENO R, OYARZÚN M. Recomendaciones sobre informe espirométrico. Segunda parte. Enf Respir Cir Torác 1988; 4: 138-49.

15.- KNUDSON R, LEBOWITZ M, HOLBERG C, BURROWS B. Changes in the Normal Maximal Expiratory Flow-Volumen Curve with Growth and Aging. Am Rev Respir Dis 1983; 127: 725-34.

16.- GUTIÉRREZ M,CARVAJAL M, DEL FIERRO A. Comparación de dos criterios de informe espirométrico. Enf Respir Cir Torác 1987; 3 (Supl): 23.

17.- GUTIÉRREZ M, ROJAS A, RIOSECO F, DEL FIERRO A, LINDERMAN C, MEYER G, VARELA C. Valores Espirométricos en chilenos entre 5 y 19 años a nivel del mar. Enf Respir Cir Torác 1990; 6 (Supl): 39.

18.- CORRALES R, DEL FIERRO A, GUTIÉRREZ M, LEIVA A, LINDERMAN C, MYER G, et al. Valores espirométricos normales para niños chilenos. Rev Enf Respir Cir Torác 1992; 8: 148-57.

19.- MARAMBIO J A, SANDOVAL H, VALENZUELA P, MORENO R, DONOSO H, CONTRERAS G, RETAMALES E. Espirometría en población general del Gran Santiago: Comparación con los valores de referencia de Knudson. Rev Chil Enf Respir 1992; 8: 241.

20.- GUTIÉRREZ M, RIOSECO F, ROJAS A, CASANOVA D. Determinación de valores espirométricos en una población chilena normal mayor de 5 años, a nivel del mar. Rev Med Chile 1996; 124: 1295-306.

21.- GUTIÉRREZ M, RIOSECO F, ROJAS A, CASANOVA D. Ecuaciones de referencia espirométricas en población chilena. Rev Chil Enf Respir 1997; 13: 165-77.

22.- GONZÁLEZ P, UNDURRGA A, CHOMALY M, VIVANCO S. Prevalencia de alteraciones espirométricas en adultos consultantes generales de atención primaria. Comparación de resultados según valores de referencia nacionales y extranjeros. Rev Chil Enf Respir 1999; 15: 258.

23.- DÍAZ O, VILLAFRANCA C, LEIVA A, LISBOA C. Evaluación clínico funcional en la EPOC de acuerdo a Knudson y Gutiérrez. Rev Chil Enf Respir. 1998; 14: 216.

24.- PALACIOS S, MARTÍNEZ A, FLORES G. Comparación de valores de referencia foráneos y nacionales en el análisis de índices espirométricos en pacientes con estenosis mitral cerrada. Rev Chil Enf Respir 1998; 14: 215.

25.- Standardization of Spirometry, 1994 Update. American Thoracic Society. Am J Respir Crit Care Med 1994; 152: 1107-36.

26.- PÉREZ-PADILLA R, VALDIVIA G., MENEZES A. Valores de referencia espirométrica en cinco grandes ciudades de Latinoamérica para sujetos de 40 o más años de edad.[PLATINO] (En prensa).

27.- Official Statement of the European Respiratory Society. Standardized Lung Function Testing. Update 1993. Eur Respir J 1993; 6 (Suppl): 16.

28.- PAUKER S G, KOPELMAN R I. Interpreting hoofbeats: can Bayes help clear the haze? N Engl J Med 1992; 327: 1009-13.

29.- American Thoracic Society. Lung function testing: selection of reference values and interpretative strate- 
gies. Am Rev Respir Dis 1991; 144: 1202-18.

30.- PALACIOS S, MARTÍNEZ A, FLORES G, PÉREZ O, OLMOS A. Validación de los valores de referencia espirométricos nacionales. Rev Chil Enf Respir 1998; 14: 215.

31.- ROJAS N, PLATERO A, QUIJADA D, STEVENS J, DUSSAUBAT N, OYARZÚN M. Comparación de valores espirométricos de referencia en una muestra de estudiantes de medicina. Rev Chil Enf Respir 2004; 20: 241.

32.- GUTIÉRREZ M. Comparación de valores espirométricos chilenos con valores de referencia para población mejicano-americana de Estados Unidos. Rev Chil Enf Respir 2001; 17: 267.
33.- SWANNEY M P, BECKERT L E, FRAMPTON C M, WALLACE L A, JENSEN R L, CRAPO R O. Validity of the American Thoracic Society and other spirometric algorithms using FVC and forced expiratory volume at $6 \mathrm{~s}$ for predicting a reduced total lung capacity. Chest 2004; 126: 1861-6.

34.- AARÓN S D, DALES R E, CARDINAL P. How accurate is spirometry at predicting restrictive pulmonary impairment? Chest 1999; 115: 86973.

35.- GLADY C A, AARÓN S D, LUNAU M, CLINCH J, DALES R E. A spirometry based algorithm to direct lung function testing in the pulmonary function laboratory. Chest 2003; 123: 1939-46. 